\title{
Optimal Expected Utility of Wealth for Two Dependent Classes of Insurance Business
}

\author{
Cristina Gosio, Ester C. Lari, Marina Ravera \\ Department of Economics and Business, University of Genova, Genova, Italy \\ Email: gosio@economia.unige.it, lari@economia.unige.it, ravera@economia.unige.it
}

Received February 18, 2013; revised March 18, 2013; accepted April 12, 2013

Copyright (C) 2013 Cristina Gosio et al. This is an open access article distributed under the Creative Commons Attribution License, which permits unrestricted use, distribution, and reproduction in any medium, provided the original work is properly cited.

\begin{abstract}
We consider a modified version of the classical Cramer-Lundberg risk model. In particular, we assume two classes of insurance business dependent through the claim number process $N_{i}, i=1,2$ : we consider that the number of claims is generated by a bivariate Poisson distribution $\left(N_{1}, N_{2}\right)$. We also consider the presence of a particular kind of reinsurance contract, supposing that the first insurer concludes an Excess of Loss reinsurance limited by $L_{i}, i=1,2$, with retention limits $b_{i}, i=1,2$, for the respective classes of insurance business. The aim of this paper is to maximize the expected utility of the wealth of the first insurer, having the retention limits as decision variables. We assume an exponential utility function and, fixed $L_{i}, i=1,2$, we discuss optimal $b_{i}, i=1,2$.
\end{abstract}

Keywords: Bivariate Poisson Distribution; Excess of Loss; Exponential Utility Function; Reinsurance; Retention Limits; Risk Theory

\section{Introduction}

The classical Cramer-Lundberg risk model assumes that the number of claims up to time $t$ is independent of the claim size $X_{K}$ and the claim sizes are independent and identically distributed. However, the independence assumption can be restrictive in practical applications. Several authors have therefore suggested models where the risks dependence is assumed. Within this models, we distinguish between risk models with dependence among claim size and among inter-occurrence time (see [1-5]) and risk models that consider aggregate claims amount processes generated by correlated classes of insurance business (see [6-13]). In [14] a wide set of dependent risk processes involving particular classes is presented with references therein. In some cases, the authors consider reinsurance contracts (see $[9,10]$ ) to improve the risk situation to which the company is subjected. In particular, [10] suggests an unlimited Excess of Loss reinsurance and maximizes the expected utility of the wealth of the insurer or the adjustment coefficient. In this paper, we consider a risk model involving two dependent classes of insurance business and a limited Excess of Loss reinsurance with the aim to maximize the expected utility of the first insurer wealth, having the retention limits as deci- sion variables. The paper is organized as follows. Section 2 presents the risk model and the reinsurance contract. In Section 3 the maximization problem is proposed and in Section 4 the solution is discussed.

\section{The Model}

We consider a risk model involving two dependent classes of insurance business. Let $X_{1, j}$ be the claim size random variables for the first class with common distribution function $F_{1}$ and let $X_{2, j}$ be those for the second class with common distribution function $F_{2}$. We assume that $F_{1}$ and $F_{2}$ have continuous and positive first derivative, with $F_{i}(x)=0$ if $x \leq 0, i=1,2$. Their expected values are denoted by $\mu_{i}<+\infty, i=1,2$, respectively. Then, the aggregate claims amount process generated from the two classes of business, in a given period of time, is $S=S_{1}+S_{2}$ with

$$
S_{i}=\sum_{j=1}^{N_{i}} X_{i j}, i=1,2,
$$

where $N_{i}, i=1,2$, is the number of claims, for classes $i=1,2$, in the given period of time.

We assume that $\left\{X_{1, j}, j=1,2, \cdots\right\}$ and $\left\{X_{2, j}, j=1,2, \cdots\right\}$ are independent, and they are inde- 
pendent of $N_{1}$ and $N_{2}$. The claim number processes are correlated by means of the following relationships (see [10]):

$$
N_{1}=K_{1}+K \text { and } N_{2}=K_{2}+K
$$

where $K_{1}, K_{2}$ and $K$ are independent Poisson random variables with parameters $\alpha_{1}, \alpha_{2}$ and $\alpha$, respectively.

As usual, we define the surplus process in the given period of time:

$$
U=u+c_{1}+c_{2}-\left(S_{1}+S_{2}\right)
$$

where $c_{i}, i=1,2$, is the insurance premium of risk $i$ and $u$ is the value of the surplus at the beginning of the time period.

We assume the following exponential utility function:

$$
u(x)=-\mathrm{e}^{-\beta x}, \beta>0
$$

then, in the absence of reinsurance, the expected utility of wealth is

$$
E\left[-\mathrm{e}^{\left\{-\beta\left[u+c_{1}+c_{2}-\left(S_{1}+S_{2}\right)\right]\right\}}\right]
$$

We instead assume that the first insurer concludes an Excess of Loss (XL) reinsurance contract limited by $L_{i}, i=1,2$, with retention limits $b_{i}, i=1,2$, for the respective classes of insurance business. In [10], an unlimited XL reinsurance is considered.

In the following, we will consider the variables $X_{i}, i=1,2$, identically distributed to $X_{i, j}, i=1,2$. In practical, XL reinsurance contracts with retention limit $b_{i}>0$, are limited by some constant $L_{i}, 0<L_{i} \leq+\infty$, which leads to the following division of claim size $X_{i}$. The reinsurer pays $Y_{i}\left(b_{i}, L_{i}\right)=\min \left\{\max \left\{X_{i}-b_{i}, 0\right\}, L_{i}\right\}$ and the first insurer pays what is left: $X_{i}\left(b_{i}, L_{i}\right)=X_{i}-Y_{i}\left(b_{i}, L_{i}\right)$, (see [15]). That is:

$$
\begin{aligned}
& Y_{i}\left(b_{i}, L_{i}\right) \\
& = \begin{cases}0, & \text { if } X_{i} \leq b_{i} \\
X_{i}-b_{i}, & \text { if } b_{i}<X_{i} \leq b_{i}+L_{i}, i=1,2 \\
L_{i}, & \text { if } X_{i}>b_{i}+L_{i}\end{cases}
\end{aligned}
$$

and

$$
\begin{aligned}
& X_{i}\left(b_{i}, L_{i}\right) \\
& = \begin{cases}X_{i}, & \text { if } X_{i} \leq b_{i} \\
b_{i}, & \text { if } b_{i}<X_{i} \leq b_{i}+L_{i} \\
X_{i}-L_{i}, & \text { if } X_{i}>b_{i}+L_{i}, \quad i=1,2\end{cases}
\end{aligned}
$$

Observe that if $L_{i}=+\infty$ the limited XL reinsurance becomes unlimited. We fix $L_{i}, i=1,2$, with $0<L_{i}<+\infty$, for the two classes of insurance business, respectively, and we make use of the retention limits $b_{i}, i=1,2$, as decision variables. We refer to (6) and (7) and we set

$$
Y_{i}\left(b_{i}, L_{i}\right)=\bar{Y}_{i}\left(b_{i}\right), i=1,2,
$$

identically distributed to the $j$-th payment of the reinsurer $\bar{Y}_{i j}\left(b_{i}\right), j=1,2, \cdots$ and

$$
X_{i}\left(b_{i}, L_{i}\right)=\bar{X}_{i}\left(b_{i}\right), i=1,2,
$$

identically distributed to the $j$-th payment of the first insurer $\bar{X}_{i j}\left(b_{i}\right), j=1,2, \cdots$.

Then, the aggregate claims amount process for the insurer generated from the two classes of business after reinsurance, in a given period of time, is $S_{1}\left(b_{1}\right)+S_{2}\left(b_{2}\right)$ with

$$
S_{i}\left(b_{i}\right)=\sum_{j=1}^{N_{i}} \bar{X}_{i j}\left(b_{i}\right), i=1,2 .
$$

As usually, the reinsurance premiums are evaluated as follows:

$$
c_{i}\left(b_{i}\right)=\left(1+\theta_{i}\right) E\left[N_{i}\right] E\left[\bar{Y}_{i}\left(b_{i}\right)\right], i=1,2,
$$

where $\theta_{i}>0, i=1,2$, is the corresponding safety loading. We therefore have

$$
\begin{aligned}
& c_{i}\left(b_{i}\right) \\
& =\left(1+\theta_{i}\right)\left(\alpha_{i}+\alpha\right) \int_{b_{i}}^{b_{i}+L_{i}}\left[1-F_{i}(x)\right] \mathrm{d} x, i=1,2 .
\end{aligned}
$$

Then, after reinsurance, the surplus process in the given period of time is

$$
\begin{aligned}
& \bar{U}\left(b_{1}, b_{2}\right) \\
& =u+c_{1}+c_{2}-\left(c_{1}\left(b_{1}\right)+c_{2}\left(b_{2}\right)\right)-\left(S_{1}\left(b_{1}\right)+S_{2}\left(b_{2}\right)\right)
\end{aligned}
$$

and the expected utility is

$$
\begin{aligned}
& E\left[-\mathrm{e}^{\left\{-\beta \bar{U}\left(b_{1}, b_{2}\right)\right\}}\right] \\
& =\mathrm{e}^{-\beta\left(u+c_{1}+c_{2}\right)} \mathrm{e}^{\beta\left(c_{1}\left(b_{1}\right)+c_{2}\left(b_{2}\right)\right)} E\left[-\mathrm{e}^{\beta\left(S_{1}\left(b_{1}\right)+S_{2}\left(b_{2}\right)\right)}\right] .
\end{aligned}
$$

In the following Section, we will take the problem of the maximization of the wealth expected utility, having the retention limits $b_{i}, i=1,2$, as decision variables.

\section{The Problem}

As already mentioned, our problem is to find the pair $\left(b_{1}, b_{2}\right)$, with $b_{i} \geq 0, i=1,2$, that maximizes (13). We observe that from the moment generating function of the bivariate Poisson distribution (see $[10,16]$ ) it follows that

$$
\begin{aligned}
E & {\left[\mathrm{e}^{\beta\left(S_{1}\left(b_{1}\right)+S_{2}\left(b_{2}\right)\right)}\right] } \\
= & \mathrm{e}^{\left\{\left(\alpha_{1}+\alpha\right)\left(M_{\bar{X}_{1}\left(b_{1}\right)}(\beta)-1\right)+\left(\alpha_{2}+\alpha\right)\left(M_{\bar{X}_{2}\left(b_{2}\right)}(\beta)-1\right)\right\}} \\
& \times \mathrm{e}^{\left\{\alpha\left(M_{\bar{X}_{1}\left(b_{1}\right)}(\beta)-1\right)\left(M_{\bar{X}_{2}\left(b_{2}\right)}(\beta)-1\right)\right\}}
\end{aligned}
$$

where we assume that the moment generating function 
$M_{\bar{X}_{i}\left(b_{i}\right)}(\beta), i=1,2$, exists. In particular, we assume that the variables $X_{i}$ have a limited distribution or that the following relationship is true:

$$
\begin{aligned}
& \lim _{x \rightarrow+\infty} \mathrm{e}^{\beta x}\left[1-F_{i}(x)\right]=0, \\
& i=1,2
\end{aligned}
$$

It therefore results

$$
\begin{aligned}
& M_{\bar{X}_{i}\left(b_{i}\right)}(\beta) \\
& =E\left[\mathrm{e}^{\left.\beta \bar{X}_{i}\left(b_{i}\right)\right]}\right] \\
& =\int_{0}^{b_{i}} \mathrm{e}^{\beta x} \mathrm{~d} F_{i}(x)+\mathrm{e}^{\beta b_{i}} \operatorname{Prob}\left[b_{i}<X_{i} \leq b_{i}+L_{i}\right] \\
& \quad+\int_{b_{i}+L_{i}}^{\infty} \mathrm{e}^{\beta\left(x-L_{i}\right)} \mathrm{d} F_{i}(x) \\
& =1+\beta\left\{\int_{0}^{b_{i}} \mathrm{e}^{\beta x}\left[1-F_{i}(x)\right] \mathrm{d} x+\mathrm{e}^{-\beta L_{i}} \int_{b_{i}+L_{i}}^{\infty} \mathrm{e}^{\beta x}\left[1-F_{i}(x)\right] \mathrm{d} x\right\} \\
& i=1,2
\end{aligned}
$$

We put:

$$
\begin{aligned}
& \int_{0}^{b_{i}} \mathrm{e}^{\beta x}\left[1-F_{i}(x)\right] \mathrm{d} x+\mathrm{e}^{-\beta L_{i}} \int_{b_{i}+L_{i}}^{\infty} \mathrm{e}^{\beta x}\left[1-F_{i}(x)\right] \mathrm{d} x \\
& =B_{i}\left(b_{i}\right), \\
& i=1,2 .
\end{aligned}
$$

and we straightaway observe that

$$
B_{i}^{\prime}\left(b_{i}\right)=\mathrm{e}^{\beta b_{i}}\left[F_{i}\left(b_{i}+L_{i}\right)-F_{i}\left(b_{i}\right)\right]>0, i=1,2
$$

since it is $L_{i}>0$ and $F_{i}^{\prime}(x)>0$ by assumption.

Because of (14), (16) and (18), (13) can be written as

$$
\begin{aligned}
E\left[\mathrm{e}^{\left\{-\beta \bar{U}\left(b_{1}, b_{2}\right)\right\}}\right] & \\
=\mathrm{e}^{-\beta\left(u+c_{1}+c_{2}\right)} \times\{ & -\mathrm{e}^{\beta\left(c_{1}\left(b_{1}\right)+c_{2}\left(b_{2}\right)+\left(\alpha_{1}+\alpha\right) B_{1}\left(b_{1}\right)\right)} \\
& \left.\times \mathrm{e}^{\beta\left(\left(\alpha_{2}+\alpha\right) B_{2}\left(b_{2}\right)+\alpha \beta B_{1}\left(b_{1}\right) B_{2}\left(b_{2}\right)\right)}\right\}
\end{aligned}
$$

that is, putting

$$
\begin{aligned}
& c_{1}\left(b_{1}\right)+c_{2}\left(b_{2}\right)+\left(\alpha_{1}+\alpha\right) B_{1}\left(b_{1}\right)+\left(\alpha_{2}+\alpha\right) B_{2}\left(b_{2}\right) \\
& +\alpha \beta B_{1}\left(b_{1}\right) B_{2}\left(b_{2}\right)=H\left(b_{1}, b_{2}\right),
\end{aligned}
$$

we have

$$
\begin{aligned}
& E\left[\mathrm{e}^{\left\{-\beta \bar{U}\left(b_{1}, b_{2}\right)\right\}}\right] \\
& =\mathrm{e}^{-\beta\left(u+c_{1}+c_{2}\right)}\left\{-\mathrm{e}^{\beta H\left(b_{1}, b_{2}\right)}\right\} .
\end{aligned}
$$

Therefore, maximizing (13) is equivalent to minimizing $H\left(b_{1}, b_{2}\right)$; it follows that, since $\min \left\{H\left(b_{1}, b_{2}\right)\right\}=-\max \left\{-H\left(b_{1}, b_{2}\right)\right\}$, our problem consists of

$$
P:\left\{\begin{array}{l}
\max _{b_{1}, b_{2}}\left\{-H\left(b_{1}, b_{2}\right)\right\} \\
b_{1} \geq 0 \\
b_{2} \geq 0 .
\end{array}\right.
$$

We proceed in a similar way to that followed in [10]. The Kuhn-Tucker conditions for the $P$ problem are

$$
\left\{\begin{array}{l}
\frac{\partial H}{\partial b_{i}}=\lambda_{i} \\
\lambda_{i} \geq 0 \\
b_{i} \geq 0 \\
\lambda_{i} b_{i}=0
\end{array}, \forall i=1,2,\right.
$$

where, remembering (11) and (18):

$$
\begin{aligned}
& \frac{\partial H}{\partial b_{1}} \\
= & {\left[F_{1}\left(b_{1}+L_{1}\right)-F_{1}\left(b_{1}\right)\right] } \\
& \times\left\{-\left(1+\theta_{1}\right)\left(\alpha_{1}+\alpha\right)+\mathrm{e}^{\beta B_{1}}\left[\left(\alpha_{1}+\alpha\right)+\alpha \beta B_{2}\left(b_{2}\right)\right]\right\}
\end{aligned}
$$

and

$$
\begin{aligned}
\frac{\partial H}{\partial b_{2}}=[ & \left.F_{2}\left(b_{2}+L_{2}\right)-F_{2}\left(b_{2}\right)\right] \\
\times & \left\{-\left(1+\theta_{2}\right)\left(\alpha_{2}+\alpha\right)\right. \\
& \left.+\mathrm{e}^{\beta b_{2}}\left[\left(\alpha_{2}+\alpha\right)+\alpha \beta B_{1}\left(b_{1}\right)\right]\right\}
\end{aligned}
$$

Let us start by proving the following theorem.

\section{Theorem 1.}

The Hessian matrix of $-H\left(b_{1}, b_{2}\right)$ is negative definite, whenever the gradient is null.

Proof. Considering (23) and (24), it results

$$
\begin{aligned}
& \left.\frac{\partial^{2}(-H)}{\partial b_{1}^{2}}\right|_{\frac{\partial H}{\partial b_{1}}=0}=-\beta \mathrm{e}^{\beta b_{1}}\left[F_{1}\left(b_{1}+L_{1}\right)-F_{1}\left(b_{1}\right)\right] \\
& \times\left\{\alpha_{1}+\alpha\left[1+\beta B_{2}\left(b_{2}\right)\right]\right\}<0 \\
& \left.\frac{\partial^{2}(-H)}{\partial b_{2}^{2}}\right|_{\frac{\partial H}{\partial b_{2}}=0}=-\beta \mathrm{e}^{\beta b_{2}}\left[F_{2}\left(b_{2}+L_{2}\right)-F_{2}\left(b_{2}\right)\right] \\
& \times\left\{\alpha_{2}+\alpha\left[1+\beta B_{1}\left(b_{1}\right)\right]\right\}<0 \\
& \left.\frac{\partial^{2}(-H)}{\partial b_{1} \partial b_{2}}\right|_{\frac{\partial H}{\partial b_{1}}=\frac{\partial H}{\partial b_{2}}=0}=-\left[F_{1}\left(b_{1}+L_{1}\right)-F_{1}\left(b_{1}\right)\right] \\
& \times\left[F_{2}\left(b_{2}+L_{2}\right)-F_{2}\left(b_{2}\right)\right] \alpha \beta \mathrm{e}^{\beta\left(b_{1}+b_{2}\right)}
\end{aligned}
$$

and 


$$
\begin{aligned}
& \frac{\partial^{2}(-H)}{\partial b_{1}^{2}} \frac{\partial^{2}(-H)}{\partial b_{2}^{2}}-\left.\left(\frac{\partial^{2}(-H)}{\partial b_{1} \partial b_{2}}\right)^{2}\right|_{\frac{\partial H}{\partial b_{1}}=\frac{\partial H}{\partial b_{2}}=0} \\
= & \beta^{2} \mathrm{e}^{\beta\left(b_{1}+b_{2}\right)}\left[F_{1}\left(b_{1}+L_{1}\right)-F_{1}\left(b_{1}\right)\right] \times\left[F_{2}\left(b_{2}+L_{2}\right)-F_{2}\left(b_{2}\right)\right] \\
& \times\left\{\alpha_{1} \alpha_{2}+\left[\alpha_{1}\left(1+\beta B_{1}\left(b_{1}\right)\right)+\alpha_{2}\left(1+\beta B_{2}\left(b_{2}\right)\right)\right] \alpha\right. \\
& \left.+\left\{\left[1+\beta B_{2}\left(b_{2}\right)\right]\left[1+\beta B_{1}\left(b_{1}\right)\right]-\left[F_{1}\left(b_{1}+L_{1}\right)-F_{1}\left(b_{1}\right)\right]\left[F_{2}\left(b_{2}+L_{2}\right)-F_{2}\left(b_{2}\right)\right] \cdot \mathrm{e}^{\beta\left(b_{1}+b_{2}\right)}\right\} \alpha^{2}\right\} \\
= & \beta^{2} \mathrm{e}^{\beta\left(b_{1}+b_{2}\right)}\left[F_{1}\left(b_{1}+L_{1}\right)-F_{1}\left(b_{1}\right)\right]\left[F_{2}\left(b_{2}+L_{2}\right)-F_{2}\left(b_{2}\right)\right] \\
& \times\left\{\alpha_{1} \alpha_{2}+\left[\alpha_{1}\left(1+\beta B_{1}\left(b_{1}\right)\right)+\alpha_{2}\left(1+\beta B_{2}\left(b_{2}\right)\right)\right] \alpha\right. \\
& \left.+\left[\mathrm{e}^{\beta b_{1}}\left[F_{1}\left(b_{1}+L_{1}\right)-F_{1}\left(b_{1}\right)\right] Q_{2}\left(b_{2}\right)+\mathrm{e}^{\beta b_{2}}\left[F_{2}\left(b_{2}+L_{2}\right)-F_{2}\left(b_{2}\right)\right] Q_{1}\left(b_{1}\right)+Q_{1}\left(b_{1}\right) Q_{2}\left(b_{2}\right)\right] \alpha^{2}\right\} \\
>0 &
\end{aligned}
$$

where

$$
\begin{aligned}
& Q_{i}\left(b_{i}\right)=\int_{0}^{b_{i}} \mathrm{e}^{\beta x} \mathrm{~d} F_{i}(x)+\int_{b_{i}+L_{i}}^{\infty} \mathrm{e}^{\beta x} \mathrm{~d} F_{i}(x), \\
& i=1,2 .
\end{aligned}
$$

\section{The Khun-Tucker Conditions}

We look for the points $\left(b_{1}, b_{2}\right)$ that fulfill the KuhnTucker conditions. To this purpose, we put

$$
\begin{gathered}
\frac{\alpha \beta B_{1}(0)}{\alpha_{2}+\alpha}=a_{1} \text { and } \frac{\alpha \beta B_{2}(0)}{\alpha_{1}+\alpha}=a_{2} \\
\frac{1}{\beta} \ln \frac{1+\theta_{1}}{1+a_{2}}=\bar{b}_{1} \text { and } \frac{1}{\beta} \ln \frac{1+\theta_{2}}{1+a_{1}}=\bar{b}_{2} \\
\frac{\alpha \beta B_{1}\left(\bar{b}_{1}\right)}{\alpha_{2}+\alpha}=A_{1} \text { and } \frac{\alpha \beta B_{2}\left(\bar{b}_{2}\right)}{\alpha_{1}+\alpha}=A_{2}
\end{gathered}
$$

and we prove the following theorem where $a_{i}, \bar{b}_{i}$ and $A_{i}, i=1,2$, are defined by (25), (26) and (27), respectively.

\section{Theorem 2.}

1) The point $(0,0)$ satisfies the system (22) if and only if it is

$$
\theta_{1} \leq a_{2} \text { and } \theta_{2} \leq a_{1} \text {. }
$$

2) The point $\left(\bar{b}_{1}, 0\right)$, with $\bar{b}_{1}>0$, satisfies the system (22) if and only if it is

$$
\theta_{1}>a_{2} \text { and } \theta_{2} \leq A_{1} \text {. }
$$

3) The point $\left(0, \bar{b}_{2}\right)$, with $\bar{b}_{2}>0$, satisfies the system (22) if and only if it is

$$
\theta_{1} \leq A_{2} \text { and } \theta_{2}>a_{1} \text {. }
$$

4) If it is

$$
\theta_{1}>a_{2} \text { and } \theta_{2}>a_{1}
$$

and it is

$$
\theta_{1}>A_{2} \text { and } \theta_{2}>A_{1}
$$

or

$$
\theta_{1}<A_{2} \text { and } \theta_{2}<A_{1}
$$

then it exists the point $\left(\hat{b}_{1}, \hat{b}_{2}\right)$, with $0<\hat{b}_{1}<\bar{b}_{1}$ and $0<\hat{b}_{2}<\bar{b}_{2}$, satisfying the system (22), and it is

$$
\left\{\begin{array}{l}
\hat{b}_{1}=\frac{1}{\beta} \ln \frac{1+\theta_{1}}{1+\frac{\alpha \beta B_{2}\left(\hat{b}_{2}\right)}{\alpha_{1}+\alpha}} \\
\hat{b}_{2}=\frac{1}{\beta} \ln \frac{1+\theta_{2}}{1+\frac{\alpha \beta B_{1}\left(\hat{b}_{1}\right)}{\alpha_{2}+\alpha}} .
\end{array}\right.
$$

Proof.

1) It results

$$
\begin{aligned}
& \left.\frac{\partial H}{\partial b_{1}}\right|_{b_{1}=b_{2}=0} \\
& =F_{1}\left(L_{1}\right)\left\{-\theta_{1}\left(\alpha_{1}+\alpha\right)+\alpha \beta B_{2}(0)\right\} \geq 0 \Leftrightarrow
\end{aligned}
$$

the first of (28) holds and

$$
\begin{aligned}
& \left.\frac{\partial H}{\partial b_{2}}\right|_{b_{1}=b_{2}=0} \\
& =F_{2}\left(L_{2}\right)\left\{-\theta_{2}\left(\alpha_{2}+\alpha\right)+\alpha \beta B_{1}(0)\right\} \geq 0 \Leftrightarrow
\end{aligned}
$$

the second of (28) holds.

2) We recall that $\bar{b}_{1}$ is defined by (26). From (29) it follows that $\bar{b}_{1}>0 \Leftrightarrow$ the first of (29) holds.

Furthermore, remembering (25) and (26), 


$$
\begin{aligned}
& \left.\frac{\partial H}{\partial b_{1}}\right|_{b_{1}=\bar{b}_{1}, b_{2}=0} \\
= & {\left[F_{1}\left(\bar{b}_{1}+L_{1}\right)-F_{1}\left(\bar{b}_{1}\right)\right] } \\
& \times\left\{-\left(1+\theta_{1}\right)\left(\alpha_{1}+\alpha\right)+\mathrm{e}^{\beta \bar{b}_{1}}\left[\alpha_{1}+\alpha+\alpha \beta B_{2}(0)\right]\right\} \\
= & {\left[F_{1}\left(\bar{b}_{1}+L_{1}\right)-F_{1}\left(\bar{b}_{1}\right)\right] } \\
& \times\left\{-\left(1+\theta_{1}\right)\left(\alpha_{1}+\alpha\right)+\frac{1+\theta_{1}}{1+a_{2}}\left[\alpha_{1}+\alpha+\left(\alpha_{1}+\alpha\right) a_{2}\right]\right\} \\
= & 0
\end{aligned}
$$

and, remembering (27)

$$
\begin{aligned}
& \left.\frac{\partial H}{\partial b_{2}}\right|_{b_{1}=\bar{b}_{2}, b_{2}=0} \\
& =F_{2}\left(L_{2}\right)\left\{-\theta_{2}\left(\alpha_{2}+\alpha\right)+\alpha \beta B_{1}\left(\bar{b}_{1}\right)\right\} \\
& =F_{2}\left(L_{2}\right)\left\{-\theta_{2}\left(\alpha_{2}+\alpha\right)+\left(\alpha_{2}+\alpha\right) A_{1}\right\} \geq 0 \Leftrightarrow
\end{aligned}
$$

the second of (29) holds.

3) We recall that $\bar{b}_{2}$ is defined by (26). From (29) it follows that $\bar{b}_{2}>0 \Leftrightarrow$ the second of (30) holds.

Furthermore, remembering (25) and (26),

$$
\begin{aligned}
&\left.\frac{\partial H}{\partial b_{2}}\right|_{b_{1}=0, b_{2}=\bar{b}_{2}} \\
&=\left[F_{2}\left(\bar{b}_{2}+L_{2}\right)-F_{2}\left(\bar{b}_{2}\right)\right] \\
& \times\left\{-\left(1+\theta_{2}\right)\left(\alpha_{2}+\alpha\right)+\mathrm{e}^{\beta \bar{b}_{2}}\left[\alpha_{2}+\alpha+\alpha \beta B_{1}(0)\right]\right\} \\
&= {\left[F_{2}\left(\bar{b}_{2}+L_{2}\right)-F_{2}\left(\bar{b}_{2}\right)\right] } \\
& \times\left\{-\left(1+\theta_{2}\right)\left(\alpha_{2}+\alpha\right)+\frac{1+\theta_{2}}{1+a_{1}}\left[\alpha_{2}+\alpha+\left(\alpha_{2}+\alpha\right) a_{1}\right]\right\} \\
&= 0
\end{aligned}
$$

and, remembering (27)

$$
\begin{aligned}
& \left.\frac{\partial H}{\partial b_{1}}\right|_{b_{1}=0, b_{2}=\bar{b}_{2}} \\
& =F_{1}\left(L_{1}\right)\left\{-\left(1+\theta_{1}\right)\left(\alpha_{1}+\alpha\right)+\alpha \beta B_{2}\left(\bar{b}_{2}\right)\right\} \\
& =F_{1}\left(L_{1}\right)\left\{-\left(1+\theta_{1}\right)\left(\alpha_{1}+\alpha\right)+\left(\alpha_{1}+\alpha\right) A_{2}\right\} \geq 0 \Leftrightarrow
\end{aligned}
$$

the first of (30) holds.

4) Because of (31) it results

$$
\bar{b}_{1}>0 \text { and } \bar{b}_{2}>0
$$

We put

$$
\begin{aligned}
& g_{1}\left(b_{1}, b_{2}\right) \\
& =\mathrm{e}^{\beta b_{1}}\left[\left(\alpha_{1}+\alpha\right)+\alpha \beta B_{2}\left(b_{2}\right)\right]-\left(\alpha_{1}+\alpha\right)\left(1+\theta_{1}\right)
\end{aligned}
$$

and

$$
\begin{aligned}
& g_{2}\left(b_{1}, b_{2}\right) \\
& =\mathrm{e}^{\beta b_{2}}\left[\left(\alpha_{2}+\alpha\right)+\alpha \beta B_{1}\left(b_{1}\right)\right]-\left(\alpha_{2}+\alpha\right)\left(1+\theta_{2}\right)
\end{aligned}
$$

and we note that it results

$$
\left\{\begin{array} { l } 
{ \frac { \partial H } { \partial b _ { 1 } } = 0 } \\
{ \frac { \partial H } { \partial b _ { 2 } } = 0 }
\end{array} \Leftrightarrow \left\{\begin{array}{l}
g_{1}\left(b_{1}, b_{2}\right)=0 \\
g_{2}\left(b_{1}, b_{2}\right)=0 .
\end{array}\right.\right.
$$

We solve the system (35). We have:

$$
\left\{\begin{array}{l}
b_{1}=\frac{1}{\beta} \ln \frac{1+\theta_{1}}{1+\frac{\alpha \beta B_{2}\left(b_{2}\right)}{\alpha_{1}+\alpha}}=b_{1}\left(b_{2}\right) \\
g_{2}\left(b_{1}\left(b_{2}\right), b_{2}\right)=0 .
\end{array}\right.
$$

To solve the equation $g_{2}\left(b_{1}\left(b_{2}\right), b_{2}\right)=0$, we make use of a similar procedure to that in [10]. We note that $g_{2}$ is a continuous function of $b_{2}$ and that, by (34), it results

$$
\begin{aligned}
& b_{1}(0)=\bar{b}_{1}>0 \text { and } \\
& b_{1}\left(\bar{b}_{2}\right)=\frac{1}{\beta} \ln \frac{1+\theta_{1}}{1+\frac{\alpha \beta B_{2}\left(\bar{b}_{2}\right)}{\alpha_{1}+\alpha}}\left\{\begin{array}{l}
>0 \text { if }(32) \text { holds } \\
<0 \text { if }\left(32^{\prime}\right) \text { holds. }
\end{array}\right.
\end{aligned}
$$

If (32) holds, remembering (27) and (25), and being $B_{1}$ an increasing function, it results

$$
\begin{aligned}
& g_{2}\left(b_{1}(0), 0\right) \\
& =g_{2}\left(\bar{b}_{1}, 0\right) \\
& =\alpha \beta B_{1}\left(\bar{b}_{1}\right)-\theta_{2}\left(\alpha_{2}+\alpha\right) \\
& =\left(\alpha_{2}+\alpha\right)\left(A_{1}-\theta_{2}\right)<0
\end{aligned}
$$

and

$$
\begin{aligned}
& g_{2}\left(b_{1}\left(\bar{b}_{2}\right), \bar{b}_{2}\right) \\
& =\frac{1+\theta_{2}\left(\alpha_{2}+\alpha\right)}{\alpha_{2}+\alpha+\alpha \beta B_{1}(0)}\left[\left(\alpha_{2}+\alpha\right)+\alpha \beta B_{1}\left(b_{1}\left(\bar{b}_{2}\right)\right)\right] \\
& \quad-\left(\alpha_{2}+\alpha\right)\left(1+\theta_{2}\right)>0 ;
\end{aligned}
$$

it therefore exists $\hat{b}_{2}, 0<\hat{b}_{2}<\bar{b}_{2}$, satisfying the second of (36). Substituting $\hat{b}_{2}$ in (36), it results

$$
\left\{\begin{array}{l}
\hat{b}_{1}=b_{1}\left(\hat{b}_{2}\right)=\frac{1}{\beta} \ln \frac{1+\theta_{1}}{1+\frac{\alpha \beta B_{2}\left(\hat{b}_{2}\right)}{\alpha_{1}+\alpha}} \\
\hat{b}_{2}=\frac{1}{\beta} \ln \frac{1+\theta_{2}}{1+\alpha \beta B_{1}\left(b_{1}\left(\hat{b}_{2}\right)\right)}
\end{array}\right.
$$

with, by (31) and (32), since $B_{1}$ is an increasing function: 


$$
0<\frac{1}{\beta} \ln \frac{1+\theta_{1}}{1+\frac{\alpha \beta B_{2}\left(\bar{b}_{2}\right)}{\alpha_{1}+\alpha}}<\hat{b}_{1}<\frac{1}{\beta} \ln \frac{1+\theta_{1}}{1+\frac{\alpha \beta B_{2}(0)}{\alpha_{1}+\alpha}}=\bar{b}_{1}
$$

therefore, $\left(\hat{b}_{1}, \hat{b}_{2}\right)$ satisfying (33) fulfills the system (22).

Similarly, if (32') holds, remembering (27) and (25), and being $B_{1}$ an increasing function, it results

$$
g_{2}\left(b_{1}(0), 0\right)=\left(\alpha_{2}+\alpha\right)\left(A_{1}-\theta_{2}\right)>0
$$

and

$$
\begin{aligned}
& g_{2}\left(b_{1}\left(\bar{b}_{2}\right), \bar{b}_{2}\right) \\
& =\frac{1+\theta_{2}\left(\alpha_{2}+\alpha\right)}{\alpha_{2}+\alpha+\alpha \beta B_{1}(0)}\left[\left(\alpha_{2}+\alpha\right)+\alpha \beta B_{1}\left(b_{1}\left(\bar{b}_{2}\right)\right)\right] \\
& \quad-\left(\alpha_{2}+\alpha\right)\left(1+\theta_{2}\right)<0
\end{aligned}
$$

it therefore exists $\hat{b}_{2}, 0<\hat{b}_{2}<\bar{b}_{2}$, satisfying the second of (36). Substituting $\hat{b}_{2}$ in (36) we obtain (37), (38) and the point $\left(\hat{b}_{1}, \hat{b}_{2}\right)$ fulfilling the system (22).

\section{REFERENCES}

[1] H. Albrecher and O. J. Boxma, "A Ruin Model with Dependence between Claim Size and Claim Intervals," Insurance: Mathematics and Economics, Vol. 35, No. 2, 2004, pp. 245-254. doi:10.1016/j.insmatheco.2003.09.009

[2] H. Albrecher, C. Costantinescu and S. Loisel, "Explicit Ruin Formulas for Models with Dependence among Risks,” Insurance: Mathematics and Economics, Vol. 48, No. 2, 2011, pp. 265-270. doi:10.1016/j.insmatheco.2010.11.007

[3] M. Boudreault, H. Cossette, D. Landriault and E. Marceau, "On a Risk Model with Dependence between Interclaim Arrivals and Claim Sizes,” Scandinavian Actuarial Journal, No. 5, 2006, pp. 265-285. doi:10.1080/03461230600992266

[4] H. Cossette, E. Marceau and F. Marri, "Analysis of Ruin Measures for the Classical Compound Poisson Risk Model with Dependence," Scandinavian Actuarial Journal, No. 3, 2010, pp. 221-245. doi:10.1080/03461230903211992

[5] D. Landriault, "Constant Dividend Barrier in a Risk Model with Interclaim-Dependent Claim Sizes,” Insurance: $M a-$ thematics and Economics, Vol. 42, No. 1, 2008, pp. 31-
38. doi:10.1016/j.insmatheco.2006.12.002

[6] R. S. Ambagaspitiya, "On the Distribution of a Sum of Correlated Aggregate Claims,” Insurance: Mathematics and Economics, Vol. 23, No. 1, 1998, pp. 15-19. doi:10.1016/S0167-6687(98)00018-3

[7] R. S. Ambagaspitiya, "Compound Bivariate Lagrangian Poisson Distributions,” Insurance: Mathematics and Economics, Vol. 23, No. 1, 1998, pp. 21-31. doi:10.1016/S0167-6687(98)00020-1

[8] R. S. Ambagaspitiya, “Aggregate Survival Probability of a Portfolio with Dependent Subprtfolios," Insurance: Mathematics and Economics, Vol. 32, No. 3, 2003, pp. 431-443. doi:10.1016/S0167-6687(03)00131-8

[9] J. Cai and W. Wei, "Optimal Reinsurance with Positively Dependent Risks," Insurance: Mathematics and Economics, Vol. 50, No. 1, 2012, pp. 57-63. doi:10.1016/j.insmatheco.2011.10.006

[10] M. L. Centeno, "Dependent Risks and Excess of Loss Reinsurance," Insurance: Mathematics and Economics, Vol. 37, No. 2, 2005, pp. 229-238. doi:10.1016/j.insmatheco.2004.12.001

[11] G. Wang and K. C. Yuen, "On a Correlated Aggregate Claims Model with Thinning-Dependence Structure,” Insurance: Mathematics and Economics, Vol. 36, No. 3, 2005, pp. 456-468. doi:10.1016/j.insmatheco.2005.04.004

[12] K. C. Yuen, J. Guo and X. Wu, "On a Correlated Aggregate Claims Model with Poisson and Erlang Risk Processes," Insurance: Mathematics and Economics, Vol. 31, No. 2, 2002, pp. 205-214. doi:10.1016/S0167-6687(02)00150-6

[13] K. C. Yuen, J. Guo and X. Wu, "On the First Time of Ruin in the Bivariate Compound Poisson Model," Insurance: Mathematics and Economics, Vol. 38, No. 2, 2006, pp. 298-308. doi:10.1016/j.insmatheco.2005.08.011

[14] G. E. Willmot and J. K. Woo, "On the Analysis of a General Class of Dependent Risk Processes,” Insurance: Mathematics and Economics, Vol. 51, No. 1, 2012, pp. 134-141. doi:10.1016/j.insmatheco.2012.03.007

[15] C. Hipp, "Stochastic Control with Applications in Insurance," Stochastic Methods in Finance, Lecture Notes in Mathematics, Vol. 1856, 2004, pp. 127-164. doi:10.1007/978-3-540-44644-6_3

[16] L. Johnson, S. Kotz and N. Balakrishnan, "Discrete Multivariate Distributions,” Wiley, New York, 1997. 